\title{
Giardia lamblia and respiratory allergies: a study of children from an urban area with a high incidence of protozoan infections
}

\author{
Valdênia M. O. Souza, ${ }^{1}$ Iana R. F. Sales, ${ }^{2}$ Décio M. Peixoto, ${ }^{3}$

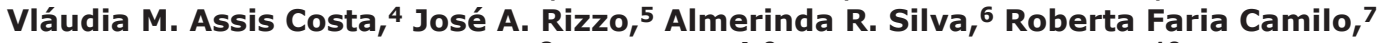 \\ Felipe Faria Pierotti, ${ }^{8}$ Dirceu Solé, ${ }^{9}$ Emanuel S. C. Sarinho ${ }^{10}$
}

\begin{abstract}
Objectives: There is a high incidence of intestinal parasite infection in urban areas in the Northeast of Brazil. Giardia lamblia infections have been associated with increased prevalence of cutaneous allergies and gastrointestinal disorders. However, little is known about the relationship between giardiasis and allergic diseases of the airways. The present study aimed to investigate the possible association between respiratory allergic diseases and infections by $G$. lamblia in children from urban areas.

Methods: This study recruited 110 patients of both sexes aged 5-15 years. Patients were administered a questionnaire evaluating clinical symptoms and were given skin tests, parasite tests and serum tests.

Results: A high incidence of $G$. lamblia was observed (45\%, 50/110). Infections by this protozoan were not associated with increased risk of respiratory allergy $(p=0.075)$, high total IgE levels $(p=0.701)$, positive specific IgE tests $(p=0.250)$, or positive skin tests for a range of environmental allergens $(p=0.239)$.

Conclusion: This study demonstrated that symptoms of asthma, skin allergy and serum markers were not associated with $\mathrm{G}$. lamblia infections in this sample of children from urban areas.
\end{abstract}

J Pediatr (Rio J). 2012;88(3):233-8: Giardia lamblia, rhinitis, asthma, skin prick test.

\section{Introduction}

Intestinal parasite infections are prevalent in developing tropical countries and are considered a severe public health problem. ${ }^{1}$ Giardia lamblia is the most commonly identified protozoan. It has an estimated mean prevalence of 2 to $5 \%$ in developed countries and 20 to $30 \%$ in emerging nations and there are approximately 280 million infections per year, leading to elevated expenditure on treatment. ${ }^{2-4}$ In underprivileged populations this infection can cause significant symptomology and further aggravate the repercussions for health of poverty and it has been put on

1. Adjunct professor, Medicina Tropical, Laboratório de Imunopatologia Keizo Asami, Universidade Federal de Pernambuco (UFPE), Recife, PE, Brazil.

2. MSc in Tropical Medicine. PhD candidate, Laboratório de Imunopatologia Keizo Asami, UFPE, Recife, PE, Brazil.

3. PhD in Pediatrics. Physician, Imunologia, Hospital das Clínicas, UFPE, Recife, PE, Brazil.

4. Adjunct professor, Laboratório de Imunopatologia Keizo Asami, UFPE, Recife, PE, Brazil.

5. Adjunct professor, Ciências da Saúde, Imunologia, Hospital das Clínicas, UFPE, Recife, PE, Brazil.

6. Assistant professor. Physician, Hospital das Clínicas, UFPE, Recife, PE, Brazil.

7. Biologist, Análises Clínicas. Master's candidate, Pediatria e Ciências Aplicadas, Universidade Federal de São Paulo (UNIFESP), São Paulo, SP, Brazil.

8. Pathology technician, UNIFESP, São Paulo, SP, Brazil.

9. Full professor, Pediatria e Ciências Aplicadas Pediatria, Imunologia Clínica e Reumatologia, UNIFESP, São Paulo, SP, Brazil.

10. PhD in Pediatrics. Associate professor, UFPE, Recife, PE, Brazil. Coordinator, Programa de Pós-graduação em Ciências da Saúde, UFPE, Recife, PE, Brazil.

No conflicts of interest declared concerning the publication of this article.

Financial support: Conselho Nacional de Desenvolvimento Científico e Tecnológico (CNPq).

Suggested citation: Souza VM, Sales IR, Peixoto DM, Costa VM, Rizzo JA, Silva AR, et al. Giardia lamblia and respiratory allergies: a study of children from an urban area with a high incidence of protozoan infections. J Pediatr (Rio J). 2012;88(3):233-8.

Manuscript submitted Nov 19 2011, accepted for publication Jan 182012.

http://dx.doi.org/10.2223/JPED.2184 
the World Health Organization's list of neglected diseases. ${ }^{5}$ The primary route of $G$. lamblia transmission is fecal-oral. ${ }^{6}$ It is a protozoan flagellate that lodges in the host intestine, where it will live commensally in the majority of cases (60$80 \%$ ). However, in some cases it can damage the mucosa and consequently cause gastrointestinal disorders, of which diarrhea with malabsorption is the most commonly reported symptom.6,7 Occasionally, associations have been reported between G. lamblia infections and urticaria and itching, ${ }^{8}$ uveitis ${ }^{9}$ and even with sensitization to food antigens, which has led to hypotheses that some type of causal nexus exists between these diseases and protozoan infections. ${ }^{7,10}$

G. lamblia releases both excretory and secretory antigens, which are the primary causes of activation of the host immunoresponse, which in turn is often characterized by a minimal inflammation of the intestinal mucosa. ${ }^{11}$ In the majority of cases this immunoresponse appears to be protective and it is primarily mediated by Immunoglobulin A (IgA) antibodies, particularly the secretory class, and by Cluster of differentiation (CD4+) T lymphocytes, which secrete and modulate a variety of cytokines. ${ }^{12,13}$ Interleukin 6 (IL-6) appears to be the most important of the cytokines for controlling acute giardiasis infection. ${ }^{14}$ Studies with mice have shown that mast cells are the principal source of IL- 6 and that animals deficient either in the cell or the cytokine react to infections by this protozoan more slowly. ${ }^{15,16}$ Other authors have pointed out that Gama Interferon (IFN- $\gamma$ ) and Interleucin 4 (IL-4) are also important, although this demonstrates that the results of this orchestration of cytokines in control of infection by G. lamblia are still under debate. ${ }^{12,17}$

There have been a growing number of studies investigating the associations between this intestinal parasite infection and allergic symptoms manifest in patients. Researchers have demonstrated that $70 \%$ of patients infected with $G$. lamblia had allergies, such as allergic rhinitis, asthma, atopic dermatitis, angioedema, acute urticaria and chronic urticaria, compared with $43 \%$ of a group free from this protozoan infection. ${ }^{7}$ Therefore, despite the fact that some of the studies conducted to date have questionable methodology, the majority have highlighted the association between Giardia infections and increased prevalence of allergic cutaneous symptoms (such as, for example, urticaria) and food allergies.7,14,18,19 In general these authors attribute the increase in these allergic parameters to changes taking place in the intestinal mucosa of the infected host, which may facilitate the absorption of inadequately metabolized protein-based antigens, resulting in the development of allergic diseases.7,20 Additionally, some studies have found that patients infected with $G$. lamblia had elevated concentrations of total and specific immunoglobulin E ( $\mathrm{IgE}$ ) and also cutaneous hyperreactivity to environmental antigens. ${ }^{7,14,19}$ On the other hand, this increase in total IgE concentrations in patients with giardiasis is debatable, since some authors only observed it when an intestinal worm infection was also present 7,19 and other studies failed to detect differences in Immunoglobulin $\mathrm{E}$ (IgE) levels between patients who were infected by $G$. lamblia and patients who were not. ${ }^{18}$

Therefore, as mentioned above, the association between G. lamblia infection and increased prevalence of symptoms of cutaneous allergies have been described with a certain frequency, but the association with respiratory tract allergies is yet to be elucidated. An elevated number of children infected with $G$. lamblia are seen at an urban pediatric clinic run by the Brazilian National Health Service (SUS - Sistema Único de Saúde) in the city of Recife and, in response to this, this study was designed to assess patients with the infection for cutaneous hypersensitivity to aeroallergens, specific IgE response and the presence of symptoms of chronic respiratory allergies.

\section{Methods}

This was a cross-sectional descriptive study that, having detected a high percentage of children and adolescents infected with $\mathrm{G}$. lamblia attempted to establish a comparative analysis of patients infected with this protozoan and those uninfected.

The study sample comprised 110 patients of both sexes, ages 5 to 15 years, recruited at a public healthcare center serving an underprivileged community in the metropolitan area of the city of Recife, PE, Brazil. Both the children and their parents or guardians agreed to participation in the study and submit to tests for G. lamblia. The selection strategy was to actively seek out children from the community who had at some time been seen for primary care, but was dependent on subjects providing specimens for the parasite tests on the days defined in the research protocol. The study began in February 2006 and ran until October 2007. Children's parents or guardians signed consent forms and all of the procedures involved in the study were approved by the Research Ethics Committee at the Universidade Federal de Pernambuco's Health Sciences Center.

The inclusion criteria for this study were: agreement to participate; having presented previously for primary care at the community health center; not having taken antiparasitic medication in the previous 12 months, availability for historytaking and clinical examination by one of the research team; and availability to provide three samples for parasite tests on alternate days. The exclusion criteria were: not meeting all inclusion criteria; children resident in the community for less than 1 year; and inability to provide information on previous history of allergic respiratory disease.

The sample size calculation was based on a priori estimates that the probability of $G$. lamblia infection was a minimum of $30 \%$ of the study sample and the prevalence of 
respiratory allergy (asthma and allergic rhinitis in isolation or combination) was around $30 \%$, based on data for Recife from the International Study of Asthma and Allergies in Childhood (ISAAC). On this basis, 48 children per group would be necessary for alpha and beta errors of 5 and $20 \%$ respectively. ${ }^{21}$

Each patient was asked to provide three stool samples, collected on alternate days and in appropriate recipients containing $10 \%$ formalin for preservation. Samples were processed using the Hoffman modified spontaneous sedimentation method, 22 which tests for the eggs or cysts of intestinal parasites. The results of the parasite tests were used to divide the sample into two groups: (A) children infected by $G$. lamblia and (B) uninfected children.

Affirmative responses to questions 2, 6 or 7 from the standardized ISAAC questionnaire 22 were defined as indicating asthma and/or allergic rhinitis and an affirmative response to question 1 was defined as indicating rhinitis. Diagnoses were confirmed by directed history-taking and clinical examination, in accordance with criteria established in consensus statements, and supplemented with the results of immediate hypersensitivity skin tests for aeroallergens.

Individuals with normal test results and no previous history of chronic respiratory disease were used for comparisons. Thus, the clinical features of respiratory allergies were classified into one of two groups for analysis: 1) respiratory allergies present; 2) free from allergic respiratory disease.

All 110 patients had immediate hypersensitivity skin tests for a range of extracts of environmental allergens Dermatophagoides pteronyssinus (DEP), Blomia tropicalis (BT), Periplaneta americana (PP) and cat epithelium (CE) -, all standardized and provided by the International Pharmaceutical Immunology - GRUPO ASAC PHARMA (IPIASAC). Histamine and saline were used as positive and negative controls respectively. The presence of papules with diameter greater than or equal to $3 \mathrm{~mm}$, with relation to the negative saline solution control, was defined as a positive reaction.

Blood was taken for total and specific IgE assays. Plasma from each patient was tested using immunofluorometric assays (ImmunoCAP System - Phadia Diagnosis) for total IgE, anti-allergen IgE (DEP, BT, PP and CE) and anti-Ascaris IgE.

Median total IgE concentrations were calculated and groups with and without parasites were analyzed and compared using the Mann-Whitney nonparametric test. Fisher's exact test was used to compare the frequencies of allergic symptoms and positive skin test and total and specific IgE test results for patients infected by G. lamblia and uninfected subjects. Results were defined as statistically significant if the statistical tests returned $p<0.05$. GraphPad Prism 3.0 was used for analyses.
The dependent variables were the symptoms of respiratory tract allergy, total $\mathrm{IgE}$ and specific IgE concentrations and immediate hypersensitivity skin tests for the common aeroallergens mentioned above. The independent variable was infection by G. lamblia. Respiratory tract allergies (allergic rhinitis and asthma, irrespective of whether symptoms were isolated or in combination) were analyzed in conjunction as chronic respiratory allergy (chronic allergic disease of the airways). Immediate hypersensitivity skin test results were defined as positive when there was a papule with a diameter greater than or equal to $3 \mathrm{~mm}$, with relation to the negative control. With regard to serum total IgE results, this variable was categorized as greater than or less than 400 IU, which is a cutoff that has been associated with atopic disease in individuals free from concurrent helminthiasis in our country. ${ }^{23}$ Specific IgE was categorized as negative or positive and results were defined as positive when over class 1 , according to the Phadia Diagnosis ImmunoCAP System standard.

\section{Results}

Out of 110 patients studied, $48 \%(n=53)$ were male and $52 \%(n=57)$ were female. Mean age was $9.5( \pm 3.2$ years). The parasite test results showed that $45 \%(n=50)$ of the entire sample was infected with intestinal parasites and $55 \%(n=60)$ were not. Among the patients with parasites, $84 \%(n=42 / 50)$ were infected by $G$. lamblia in isolation, while $16 \%(n=8)$ had coinfections, i.e., in addition to the G. lamblia infection, $8 \%(n=4)$ were also infected with Entamoeba hystolitica/Entamoeba dispar, $6 \%(n=3)$ were infected with Entamoeba coli, and 2\% $(n=1)$ had Ascaris lumbricoides parasites.

Fisher's exact test was used to evaluate associations between symptoms of asthma and infection by G. lamblia (Table 1 ), but did not detect any statistically significant differences ( $p=0.075)$, which was also the case when chronic allergic respiratory diseases were analyzed together (allergic rhinitis and/or asthma).

Table 1 also lists the proportions of infected and uninfected individuals who had elevated total IgE results and once more the difference was not statistically significant ( $p=0.701$ ). Median total IgE levels were compared using the Mann-Whitney test, but there were still no statistically significant differences between the two groups and antiAscaris IgE levels were also unable to separate the two groups, since they were low in both (Figures 1 and 2). Similarly, there was no association between specific IgE for aeroallergens and infection by $G$. lamblia (Table 1 ). Finally, there was no significant statistical difference between patients infected by $G$. lamblia and the control group in terms of positive immediate hypersensitivity skin tests results. 
Table 1 - Univariate analysis of associations between Giardia lamblia infection and clinical and laboratory variables of allergic respiratory diseases in children from a low socioeconomic status urban area in the Northeast of Brazil

\begin{tabular}{|c|c|c|c|}
\hline Variables & $\begin{array}{l}\text { Infected } \\
(n=50)\end{array}$ & $\begin{array}{l}\text { Uninfected } \\
(n=60)\end{array}$ & p* \\
\hline \multicolumn{4}{|l|}{ Clinical presentation } \\
\hline Respiratory allergy & $42(84 \%)$ & $41(68.3 \%)$ & 0.075 \\
\hline No symptoms & $8(16 \%)$ & $19(31.7 \%)$ & \\
\hline \multicolumn{4}{|l|}{ Total IgE (IU/mL) } \\
\hline$>400 \mathrm{IU}$ & $21(42 \%)$ & $23(38 \%)$ & 0.701 \\
\hline$<400 \mathrm{IU}$ & $29(58 \%)$ & $37(62 \%)$ & \\
\hline \multicolumn{4}{|l|}{ Specific IgE (IU/mL) } \\
\hline Positive & $25(50 \%)$ & $37(62 \%)$ & 0.250 \\
\hline Negative & $25(50 \%)$ & $23(38 \%)$ & \\
\hline \multicolumn{4}{|l|}{ Prick test $(\mathrm{mm})$} \\
\hline Positive & $27(54 \%)$ & $40(67 \%)$ & 0.239 \\
\hline Negative & $23(46 \%)$ & $20(33 \%)$ & \\
\hline
\end{tabular}

$\lg \mathrm{E}=$ immunoglobulin $\mathrm{E}$

* Values calculated using Fisher's exact test.

\section{Discussion}

This study was based on a sample from an underprivileged population in which a high prevalence of infections by $G$. lamblia was observed. This finding could be linked with a range of different factors, including epidemiological characteristics, since these patients come from a region where the infection is known to be endemic. Another important factor is indiscriminate use of antiparasitic drugs, since it has been reported that patients previously treated with single-dose anthelmintics, did exhibit reductions in infections by $A$. lumbricoides and $T$. trichiura, but also suffered increased infections from G. lamblia. ${ }^{24}$ The prevalence observed in this study was similar to rates that have been observed in other studies with different samples from Brazilian populations living in poor socioeconomic conditions. ${ }^{25-27}$ Although geohelminthiasis is endemic in the area studied, there was no interference from intestinal worm coinfections. The results described here appear to rule out $G$. lamblia infections having any significant influence on allergic rhinitis or asthma.

There is considerable evidence to show that parasite infections can affect allergic reactivity, especially with relation to infections by intestinal worms. ${ }^{18,28}$ Several studies investigating patients with human giardiasis have not only detected elevated immunoglobulin E levels, but also cutaneous reactions and other symptoms of allergies such as gastrointestinal allergies, which could be caused by antigens penetrating damaged mucosa.7,19 In contrast with previous investigations, we restricted our analysis to associations between giardiasis and allergies of the airways (asthma and rhinitis). The resulting data suggest that G. lamblia infections were not related to symptoms of respiratory allergies.

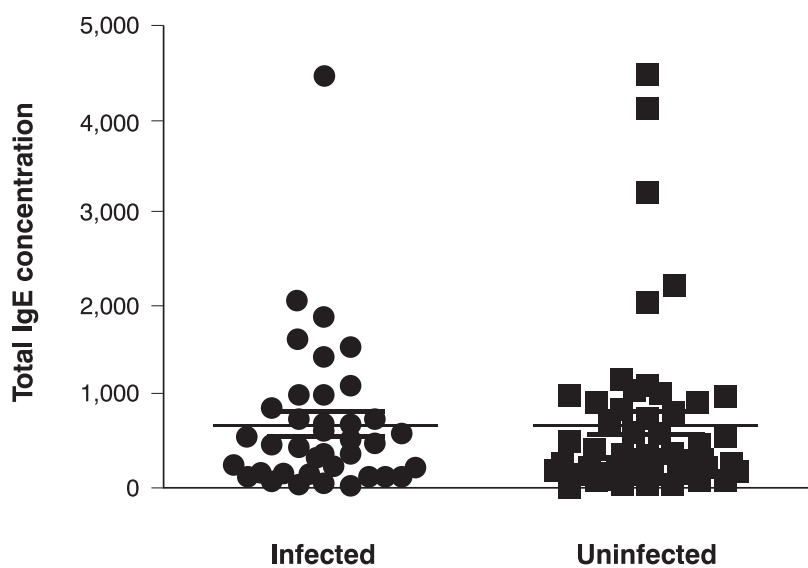

Figure 1 - One hundred and ten patients aged 5 to 15 years were tested for parasites and split into two groups: infected or not infected by G. lamblia. Blood samples were collected from members of both study groups and plasma total immunoglobulin $\mathrm{E}$ ( IgE) concentration was assayed (ImmunoCAP System - Phadia Diagnose). The Mann-Whitney test was used to analyze data expressed as median values $(p=0.8671)$ 


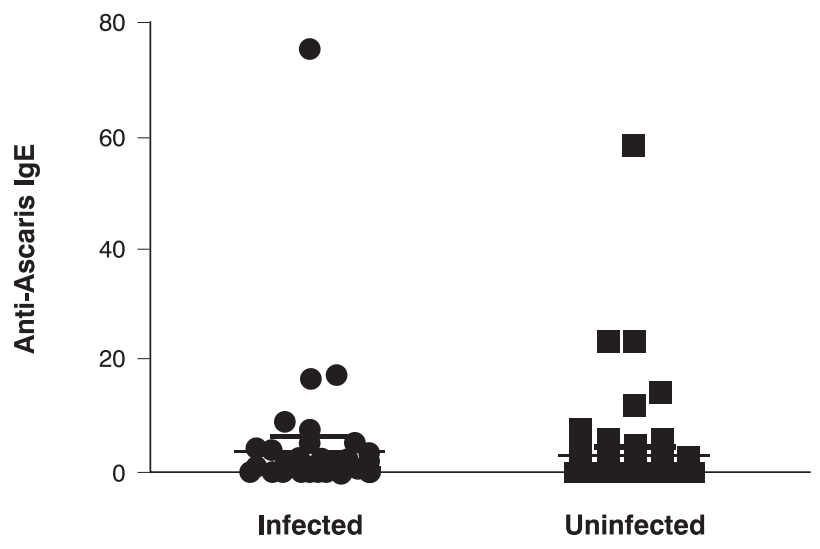

Figure 2 - One hundred and ten patients aged 5 to 15 years, were tested for parasites and split into two groups: infected or not infected by G. lamblia. Blood samples were collected from members of both study groups and plasma anti-Ascaris immunoglobulin E (IgE) concentration was assayed (ImmunoCAP System Phadia Diagnose). The Mann-Whitney test was used to analyze data $(p=0.7276)$

A study conducted by Di Prisco et al. ${ }^{7}$ found results suggestive of a possible association between $G$. lamblia and chronic respiratory disease. They found that $70 \%$ of infected patients had symptoms of allergy, including $33 \%$ with rhinitis, $14 \%$ with cutaneous allergies and $23 \%$ with asthma. That study did not exclude patients coinfected with intestinal worms, which could explain the discrepancy with relation to our results. Analyzed in conjunction, they suggest that $G$. lamblia infection could be linked with an increase in concomitant symptoms of asthma and rhinitis, not because of the protozoan infection itself, but because of concurrent helminthiasis.

We did not observe any differences between the study groups in terms of total IgE levels. Study conducted by Giacometti et al. ${ }^{18}$ also failed to detect any differences between patients infected by $G$. lamblia and uninfected patients in terms of total IgE levels, but they did find a positive association between this protozoan infection and development of cutaneous allergies. Since we only recruited patients infected by G. lamblia in isolation, we excluded patients with intestinal worm coinfections who could have had elevated total IgE levels for this reason. Similarly, an earlier study in Brazil also found normal total IgE levels in individuals infected by G. lamblia. ${ }^{29}$

Some studies have detected high levels of IgE specific for food proteins in patients infected by G. lamblia. ${ }^{7,19}$ Those findings are suggestive of an increase in gastrointestinal allergic reactivity. However, when we analyzed environmental aeroallergens, we did not observe any differences in specific IgE levels between infected and uninfected patients. ${ }^{7}$ Similarly, we did not detect any differences between the groups of patients studied here in terms of levels of IgE specific for any of the aeroallergens tested.
It has been demonstrated that there is an association between allergic symptoms and high levels of IgE anti-Ascaris and total IgE and that this is not exclusively related to geointestinal worm parasite infections. ${ }^{30,31}$ In our study, cases and controls had similarly low levels of IgE anti-Ascaris. Therefore, our results do not indicate any increased risk of developing symptoms of respiratory allergies in the presence of $G$. lamblia. There was also no association with elevated total IgE levels or even positive IgE anti-Ascaris results.

Another aspect that should be considered and which is described in the literature is that patients infected solely by G. lamblia had significantly higher TNF- $\alpha$ and rsIL-2 levels in cases of giardiasis in isolation, whereas Interleucin $1 B$ (IL-1 $\beta$ ), Interleucin 6 (IL-6) and Interleucin 8 (IL-8) were only elevated in patients who had allergies associated with the infection. ${ }^{14}$ These findings demonstrate that when allergic patients are infected by giardiasis there is increased activation of Th1 pro-inflammatory immunoresponse. Another aspect that is of relevance is the possibility of interference between giardiasis and IgA secretory function, which is known to be an important element in protecting the mucosa against protozoans and also in allergen expulsion, especially at mucosal surfaces that are exposed to greater interaction with the environment.11,13 Immunological components such as cytokines and IgA may therefore be involved in the relationship between this type of protozoan infection and respiratory allergies, preventing disease exacerbation. These features were not, however, the subject of our study.

The fact that our study did not detect a statistically significant "association" should be treated with caution since such implications can be compromised in cross-sectional studies. Nevertheless, the findings are noteworthy since the great majority of patients were exclusively infected by G. Lamblia according to all three parasite tests, in contrast with other studies.

In summary, our data suggest that G. Lamblia infections were not associated with chronic allergic diseases of the respiratory tract (concomitant asthma and/or rhinitis) or with elevated total IgE levels. It is possible that activation by protozoans of immunomodulatory mechanisms of allergic symptoms is harmful, but without clinical repercussions. However, this is conjecture and needs to be investigated, so further research on subject is needed.

\section{Acknowledgements}

This research project was supported by the Ministry of Health and the Ministry of Science and Technology (Federal Government of Brazil) and the National Council for Scientific and Technological Development (CNPq - Conselho Nacional de Desenvolvimento Científico and Tecnológico) (process number 402666/2005-4). 


\section{References}

1. World Health Organization. Control of tropical diseases. Geneva: World Health Organization; 1998.

2. Lane S, Lloyd D. Current trends in research into the waterborne parasite Giardia. Crit Rev Microbiol. 2002;28:123-47.

3. Thompson RC. Giardiasis as a re-emerging infectious disease and its zoonotic potential. Int J Parasitol. 2000;30:1259-67.

4. Rossignol JF. Cryptosporidium and Giardia: treatment options and prospects for new drugs. Exp Parasitol. 2010;124:45-53.

5. Savioli L, Smith H, Thompson A. Giardia and Cryptosporidium join the 'Neglected Diseases Initiative'. Trends Parasitol. 2006;22:203-8.

6. Robertson LJ, Hanevik K, Escobedo AA, Mørch K, Langeland N. Giardiasis - why do the symptoms sometimes never stop? Trends Parasitol. 2010;26:75-82.

7. Di Prisco MC, Hagel I, Lynch NR, Jiménez JC, Rojas R, Gil M, et al. Association between giardiasis and allergy. Ann Allergy Asthma Immunol. 1998;8:261-5.

8. Prieto-Lastra L, Pérez-Pimiento A, González-Sánchez LA, IglesiasCadarso A. Chronic urticaria and angioedema in Giardia lamblia infection. Med Clin (Barc). 2006;126:358-9.

9. Gelfer S, Scharf J, Zonis S, Mertzbach D. Acute uveitis associated with Giardia lamblia infection. Harefuah. 1984;107:75-6.

10. Hanevik K, Dizdar V, Langeland N, Hausken T. Development of functional gastrointestinal disorders after Giardia lamblia infection. BMC Gastroenterol. 2009;9:27.

11. Roxström-Lindquist $K$, Palm D, Reiner D, Ringqvist $E$, Svärd SG. Giardia immunity - an update. Trends Parasitol. $2006 ; 22: 26-31$

12. Singer SM, Nash TE. T-cell-dependent control of acute Giardia lamblia infections in mice. Infect Immun. 2000;68:170-5.

13. Langford TD, Housley MP, Boes M, Chen J, Kagnoff MF, Gillin FD, et al. Central importance of immunoglobulin $A$ in host defense against Giardia spp. Infect Immun. 2002;70:11-8.

14. Bayraktar MR, Mehmet N, Durmaz R. Serum cytokine changes in Turkish children infected with Giardia lamblia with and without allergy: effect of metronidazole treatment. Acta Trop. 2005;95:116-22

15. Zhou P, Li E, Zhu N, Robertson J, Nash T, Singer SM. Role of interleukin- 6 in the control of acute and chronic Giardia lamblia infections in mice. Infect Immun. 2003;71:1566-8.

16. Li E, Zhou P, Petrin Z, Singer SM. Mast cell-dependent control of Giardia lamblia infections in mice. Infect Immun. 2004;72:6642-9.

17. Ebert EC. Giardia induces proliferation and interferon gamma production by intestinal lymphocytes. Gut. 1999;44:342-6.

18. Giacometti A, Cirioni O, Antonicelli L, D'Amato G, Silvestri C, Del Prete MS, et al. Prevalence of intestinal parasites among individuals with allergic skin diseases. J Parasitol. 2003;89:490-2.

19. Di Prisco MC, Hagel I, Lynch NR, Barrios RM, Alvarez N, López R. Possible relationship between allergic disease and infection by Giardia lamblia. Ann Allergy. 1993;70:210-3.
20. Hardin JA, Buret AG, Olson ME, Kimm MH, Gall DG. Mast cell hyperplasia and increased macromolecular uptake in an animal model of giardiasis. J Parasitol. 1997;83:908-12.

21. Solé D, Vanna AT, Yamada E, Rizzo MC, Naspitz CK. International Study of Asthma and Allergies in Childhood (ISAAC) written questionnaire: validation of the asthma component among Brazilian children. J Investig Allergol Clin Immunol. 1998;8:376-82.

22. Hoffmann WA, Pons JA, Janer JL. The sedimentation concentration method in schistosomiasis mansoni. P R J Pub Health Trop Med. 1934;9:281-98.

23. Medeiros D, Silva AR, Rizzo JA, Motta ME, Oliveira FH, Sarinho ES. Total IgE level in respiratory allergy: study of patients at high risk for helminthic infection. J Pediatr (Rio J). 2006;82:255-9.

24. Northrop-Clewes CA, Rousham EK, Mascie-Taylor CN, Lunn PG. Anthelmintic treatment of rural Bangladeshi children: effect on host physiology, growth, and biochemical status. Am J Clin Nutr. 2001;73:53-60.

25. Teixeira JC, Heller L, Barreto ML. Giardia duodenalis infection: risk factors for children living in sub-standard settlements in Brazil. Cad Saude Publica. 2007;23:1489-93.

26. Silva RR, da Silva CA, de Jesus Pereira CA, de Carvalho Nicolato RL, Negrão-Corrêa D, Lamounier JA, et al. Association between nutritional status, environmental and socio-economic factors and Giardia lamblia infections among children aged 6-71 months in Brazil. Trans R Soc Trop Med Hyg. 2009;103:512-9.

27. Escobar-Pardo ML, de Godoy AP, Machado RS, Rodrigues D, Fagundes Neto U, Kawakami E. Prevalence of intestinal parasitoses in children at the Xingu Indian Reservation. J Pediatr (Rio J). 2010;86:493-6.

28. Carvalho EM, Bastos LS, Araújo MI. Worms and allergy. Parasite Immunol. 2006;28:525-34.

29. Geller M, Geller M, Flaherty DK, Black P, Madruga M. Serum IgE levels in giardiasis. Clin Allergy. 1978;8:69-71.

30. Takeuchi H, Zaman K, Takahashi J, Yunus M, Chowdhury HR, Arifeen $\mathrm{SE}$, et al. High titre of anti-Ascaris immunoglobulin $\mathrm{E}$ associated with bronchial asthma symptoms in 5-year-old rural Bangladeshi children. Clin Exp Allergy. 2008;38:276-82.

31. Levin ME, Le Souëf PN, Motala C. Total IgE in urban Black South African teenagers: the influence of atopy and helminth infection. Pediatr Allergy Immunol. 2008;19:449-54.

Correspondence:

Dr. Emanuel S. C. Sarinho

Centro de Pesquisa em Alergia e Imunologia,

Hospital das Clínicas

Universidade Federal de Pernambuco

CEP 50670-901 - Recife, PE - Brazil

Tel.: + 55 (81) 2126.3918

Fax: + 55 (81) 2126.3917

E-mail: emanuel.sarinho@gmail.com 